Study of hysteretic thermoelectric behavior in Título artículo / Títol article: photovoltaic materials using the finite element method, extended thermodynamics and inverse problems

Autores / Autors

Roberto Palma Guerrero, J.L. Pérez Aparicio, R. Bravo

Revista:

Energy Conversion and Management, January 2013, vol. 65

Versión / Versió:

Pre-print

Cita bibliográfica / Cita bibliogràfica (ISO 690):
PALMA GUERRERO, Roberto ; PÉREZ

APARICIO, J.L. ; BRAVO, R. Energy Conversion and Management, January 2013, vol. 65, p. 557563

url Repositori UJI:

http://hdl.handle.net/10234/91770 


\title{
Study of hysteretic thermoelectric behavior in photovoltaic materials using the finite element method, extended thermodynamics and inverse problems
}

\author{
R. Palma ${ }^{\mathrm{a}}$, J.L. Pérez-Aparicio ${ }^{\mathrm{a}}$, R. Bravo ${ }^{\mathrm{b}}$ \\ ${ }^{a}$ Mecánica de Medios Continuos y Teoría de Estructuras, Universitat Politècnica de \\ València, Spain \\ ${ }^{b}$ Structural Mechanics $\&$ Hydraulic Engineering, University of Granada, Spain
}

\begin{abstract}
The main objective of the present work is to develop and prove a theoretical explanation based on the Extended Non-Equilibrium Thermodynamics (ENET) for the hysteretical thermoelectric behavior observed in certain thinfilm photovoltaic materials. The ENET introduces dissipative fluxes in the entropy balance that could explain this behavior. To verify this explanation from a numerical point of view, results are generated using a Finite Element (FE) formulation based on the ENET and already developed in previous publications by the authors. In addition, an identification Inverse Problem (IP) is formulated; a cost function is defined as the quadratic difference between experimental and numerical results and the IP is solved minimizing the cost function using genetic algorithms. The conclusion is that the loop-like distributions are due to energy dissipation introduced by dissipative fluxes that are closely related with relaxation times. Also, the FE-IP combination permits to find an approximated characterization of properties for several materials from single experimental curves. Finally, several numerical simulations are proposed for laboratory experiments to further validate
\end{abstract}


the theoretical interpretation and to confirm the relation between relaxation times and hysteresis.

Keywords: Thin-film, Thermoelectric, Hysteresis, Finite Element Method, Extended Thermodynamics, Relaxation times, Inverse problems

\section{Introduction}

Thin-film semiconductors have drawn great attention in the last two decades due to their suitability, among other applications, for cells in solar energy. Semiconductors of the thermoelectric type are completely characterized by their figure-of-merit, that depends on thermal and electric conductivities and on the Seebeck coefficient. These properties must be determined experimentally to characterize the semiconductors and to design the solar cells themselves.

An experimental study for the measurement of the Seebeck coefficient in thin-film semiconductors has been published in [1], reporting a hysteretic behavior that prevents correct and unique measurements of this coefficient. In [2], a similar behavior was observed in the cuticle of the oriental hornet Vespa Orientalis. This cuticle seemly works as a thermoelectric heat pump for the cooling of the hornet body, and as a solar energy harvesting allowing the hornet to increase its activity in the presence of strong insolation, [3].

A theoretical and a practical challenge emerge from these works:

i) Physical interpretation of the hysteretic behavior

ii) Measurement of the Seebeck coefficient

The first was undertaken in the references, concluding that the reason for this behavior could lay in ferroelectric properties (polarization phenomena). 
Pyroelectric interactions were discussed and discarded in [1]. According to [4], the reason could be related with the heating and cooling speeds during the measurement process. For the second challenge, an empirical procedure without solid theoretical basis was proposed in [1], consisting on an analytical fitting of the experimental curves. An alternative explanation was proposed in [2], measuring the Seebeck coefficient uniquely at the heating branch.

In the present work we state that from a theoretical point of view and using the Extended Non-Equilibrium Thermodynamics (ENET) [5], hysteretic phenomena are due to the presence of multiple thermodynamic configurations accessible to the thermodynamic system; these metastable configurations are closely related with relaxation times, [6]. The ENET assumes the existence of a non-equilibrium entropy density that depends on the classical state variables and on the dissipative fluxes, in a formulation defined as mixed thermodynamic. This theory allows the study of thermodynamic systems for which the local equilibrium hypothesis is not valid, introducing relaxation times in the formulation.

We aim to undertake the two aforementioned challenges, studying them with the Finite Element (FE) formulation based on ENET developed in [7], along with classical Inverse Problem (IP) techniques. Experiments for several materials performed in [1] are numerically replicated, and in addition a Sensitivity Analysis complemented by an IP is developed. In particular the FE-IP combination is employed for the characterization of the Seebeck coefficient and, in the future, for the optimization of solar cell efficiencies. Finally, three numerical experiments for the verification of the ENET-based explanation are discussed and proposed for laboratory verification. 


\section{Theoretical formulation}

The thermoelectric balance equations are the balance of energy and of electric charge [8]:

$$
\begin{aligned}
& \rho_{m} c \dot{T}=-\nabla \cdot \boldsymbol{q}-\boldsymbol{j} \cdot \nabla V ; \\
& \nabla \cdot \boldsymbol{j}=0
\end{aligned}
$$

where $\rho_{m}, c, T, \boldsymbol{q}, \boldsymbol{j}, V$ are the mass density, heat capacity, temperature, heat flux, current density and voltage, respectively. In the previous equation the supradot $\left({ }^{\cdot}\right)$ denotes time derivative. The entropy balance required to obtain the transport equations is given by [9]:

$$
\rho_{m} \dot{s}=-\nabla \cdot \boldsymbol{j}_{s}+\sigma^{s} \Rightarrow\left\{\begin{array}{l}
\boldsymbol{j}_{s}=\frac{\boldsymbol{q}}{T} \\
\sigma^{s}=\boldsymbol{q} \cdot \nabla\left(\frac{1}{T}\right)-\frac{1}{T} \boldsymbol{j} \cdot \nabla V
\end{array}\right.
$$

where $\dot{s}, \boldsymbol{j}_{s}, \sigma^{s}$ are entropy rate, flux and production, respectively. Note that the entropy production is increased by two factors: thermal conduction and Joule heating, the latter electric energy converted into thermal one. From (2) and using the procedure described in [10], the classical transport equations are:

$$
\begin{aligned}
& \boldsymbol{q}=-\kappa \nabla T+\alpha T \boldsymbol{j} \\
& \boldsymbol{j}=-\gamma \nabla V-\alpha \gamma \nabla T
\end{aligned}
$$

where $\gamma, \kappa$ are electric and thermal conductivities and $\alpha$ the Seebeck coefficient. These parameters are usually denominated transport properties and, in general, depend on temperature as reported in [11]. 


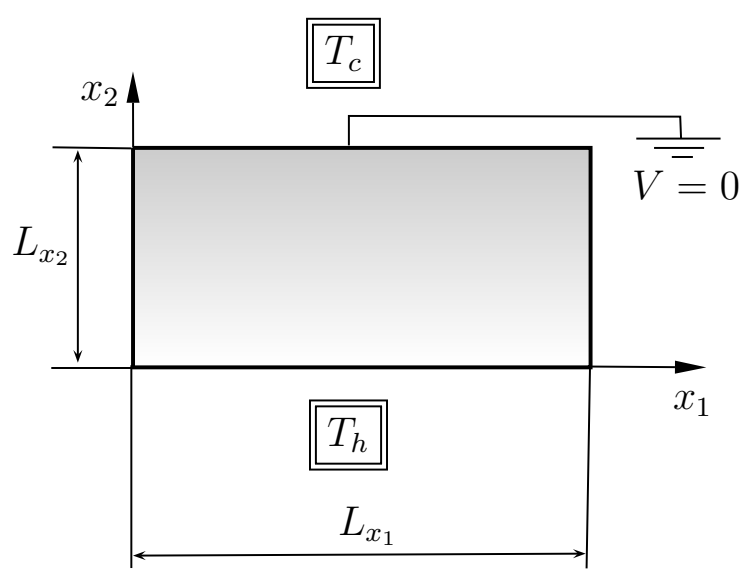

Figure 1: Experimental configuration for the measurement of the Seebeck coefficient in thermoelectric photovoltaic materials.

As discussed in the introduction, the ENET considers dissipative fluxes $\dot{\boldsymbol{q}}, \dot{\boldsymbol{j}}$ in the classical entropy balance (2). Therefore and according to [12], the entropy production becomes:

$$
\begin{aligned}
\sigma^{s}= & \boldsymbol{q} \cdot\left[\nabla\left(\frac{1}{T}\right)+\frac{C_{1}}{T} \dot{\boldsymbol{q}}+\frac{C_{3}}{T} \dot{\boldsymbol{j}}\right] \\
& +\boldsymbol{j} \cdot\left[-\frac{1}{T} \nabla V+\frac{C_{2}}{T} \dot{\boldsymbol{q}}+\frac{C_{4}}{T} \dot{\boldsymbol{j}}\right]
\end{aligned}
$$

where $C_{1}$ to $C_{4}$ are constants to be determined, closely related with relaxation times. Note that these dissipative fluxes increase the entropy, in other words, add irreversibilities to the thermodynamic system. According to [12], [7], the ENET transport equations are:

$$
\begin{aligned}
& \boldsymbol{q}=-\kappa \nabla T+\alpha T \boldsymbol{j}-\tau_{q j} \alpha T \gamma \nabla \dot{V}+\tau_{q} \kappa \nabla \dot{T} ; \\
& \boldsymbol{j}=-\gamma \nabla V-\alpha \gamma \nabla T-\tau_{j q} \alpha \gamma \nabla \dot{T}+\tau_{j} \gamma \nabla \dot{V}
\end{aligned}
$$



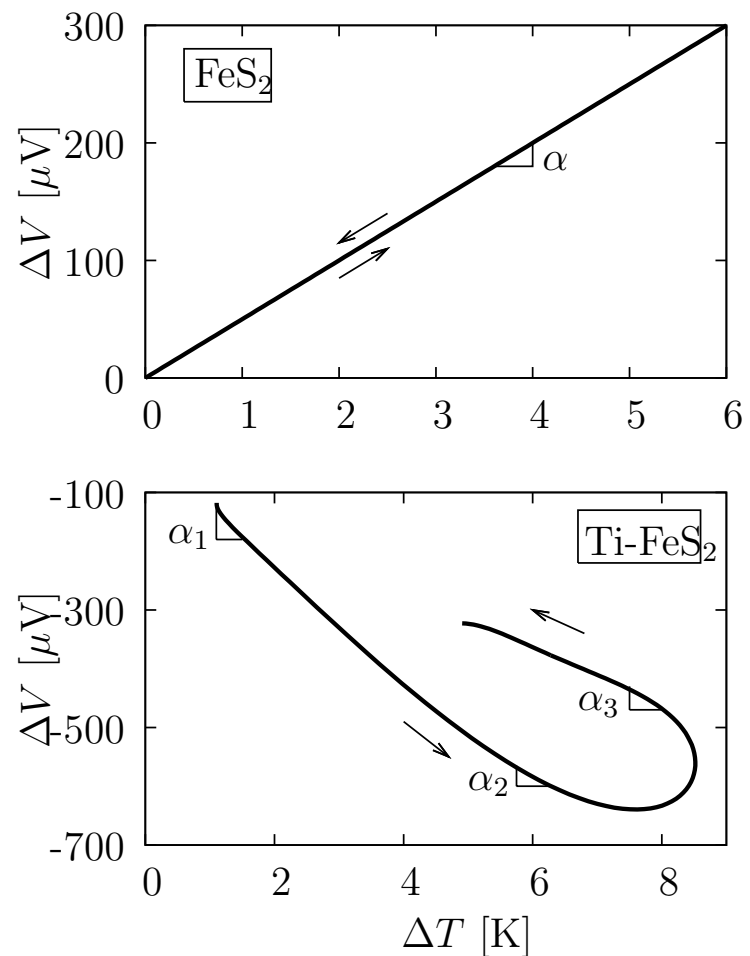

Figure 2: Experimental results reported in Ferrer 2006 [1] for $\mathrm{FeS}_{2}$ (top) and Ti-doped $\mathrm{FeS}_{2}$ (bottom) thermoelectric thin films.

The direct relaxation times $\tau_{q}, \tau_{j}$ represent thermal and electric "viscosities", closely related with Cattaneo [13], [14] and Drude [15] models; the coupling relaxation times $\tau_{q j}, \tau_{j q}$ represent thermal and electric viscosities due to the presence of voltage and of temperature gradients, respectively.

Finally, in order to obtain the thermoelectric governing equations, the boundary conditions are incorporated:

$$
\begin{array}{ll}
\text { Dirichlet }: & T=\bar{T}, \quad V=\bar{V} ; \\
\text { Neumann }: & \boldsymbol{q} \cdot \mathbf{n}=q_{c}, \quad \boldsymbol{j} \cdot \mathbf{n}=j_{c}
\end{array}
$$

where $\bar{T}, \bar{V}$ are the temperature and voltage, and $q_{c}, j_{c}$ the heat flux and 
current density, all of them prescribed. In particular, $\bar{T}$ will be $T_{h}, T_{c}$ and $\bar{V}$ the $V=0$ at the cold face in Figure 1.

\section{Physical interpretation of the hysteretic behavior}

Before developing a theoretical explanation of the hysteretic behavior using the ENET, the experimental procedure and empirical results reported in [1] are reviewed.

\subsection{Experimental procedure}

The experimental configuration for the measurement of the Seebeck coefficient usually involves thermally connecting the test device between hot $T_{h}$ and cold $T_{c}$ faces' temperature and fixing at the latter the voltage, as shown in Figure 1. Considering the classical transport equations (3) and zeroing the prescribed flux $\boldsymbol{j}$, a linear relationship between the applied temperature difference $\Delta T$ and the measured voltage drop $\Delta V$ is obtained:

$$
\left.\begin{array}{l}
\boldsymbol{j}=-\gamma \nabla V-\alpha \gamma \nabla T \\
\boldsymbol{j}=\mathbf{0}
\end{array}\right\} \Rightarrow \Delta V=-\alpha \Delta T
$$

Note that gradients have been replaced by increments to be in accordance with the experimental measurements. The Seebeck coefficient is determined plotting $\Delta V$ versus $\Delta T$ and calculating the slope $\alpha$ of the linear relationship (7); the sign of $\alpha$ is related to the semiconductor type: $\mathrm{n}$ or $\mathrm{p}$.

Two thin-film material samples with dimensions $L_{x_{2}}=15, L_{x_{1}}=25[\mathrm{~mm}]$ were characterized by the procedure described in the previous paragraph. Two very different results were observed: 
- For $\mathrm{FeS}_{2}$, the linear relationship $\Delta V-\Delta T$ shown in Figure 2 top, with constant $\alpha>0$

- For Ti-doped $\mathrm{FeS}_{2}$, the hysteretic behavior shown in Figure 2 bottom, with different values of $\alpha$

As discussed in the introduction, for samples that present a hysteretic behavior it is difficult to assign a representative Seebeck coefficient: $\alpha$ was calculated from the heating branch in [2] and from the common diagonal slope of different loops obtained applying different $\Delta T$ 's in [1], fitting the experimental results to a unique $\alpha$ :

$$
\Delta V=-\alpha \Delta T-\alpha K \frac{\mathrm{d}(\Delta T)}{\mathrm{d} t}
$$

where $K$ was an empirical magnitude with dimension of time. Therefore, the loop shape and its slope, Figure 2 (bottom), were determined by the second and first terms on the right side of (8), respectively.

\subsection{Theoretical explanation}

The motivation to provide a theoretical explanation using the ENET is due to the dependency of the empirical equation (8) on $\mathrm{d} \Delta T / \mathrm{d} t$ and on $K$. The two magnitudes can be closely related with those introduced by the ENET: dissipative fluxes and relaxation times.

The relaxation $\tau_{j}$ can be neglected in the absence of free electric charges, [7]. From the electric transport equation (5), forcing again $\boldsymbol{j}=\mathbf{0}$ :

$$
\nabla V=-\alpha \nabla T-\alpha \tau_{j q} \frac{\partial(\nabla T)}{\partial t}
$$

Comparing (8) and (9), the equivalence $K \equiv \tau_{j q}$ is evident. Therefore, the loop shape, i.e. hysteresis, is inferred to be dependent on $\tau_{j q}$. From a 
theoretical point of view, the relation between hysteresis and relaxation times already was stated in [6]: this hysteresis depends on the ratio $\tau_{j q} / t_{o b}$, where $t_{o b}$ is the observation time. The ratio depends on the material properties, observing different behaviors if:

$$
\begin{aligned}
& \tau_{j q} / t_{o b}<<1 \quad \rightarrow \quad \text { Linear response } \\
& \tau_{j q} / t_{o b} \approx 1 \quad \rightarrow \quad \text { Hysteretical response }
\end{aligned}
$$

Summarizing, from statistical physics it can be said that the microscopic relaxation time $\tau_{j q}$ increases when the $\mathrm{FeS}_{2}$ is doped with $\mathrm{Ti}$, provoking the hysterical behavior.

\section{Finite element equations}

Several numerical techniques to study the thermoelectric coupling such as the finite difference [16] and the FE methods have been published. The authors of the present work have developed several non-linear FE formulations, see [17], [18], [19], [7]. The first two are steady-state formulations; the third one is dynamic and includes the relaxation time $\tau_{q}$, permitting the study of hyperbolic propagations of temperature, voltage and heat flux after the Cattaneo model. Finally, the last work presents a complete FE formulation including three relaxation times: $\tau_{q}, \tau_{q j}, \tau_{j q}$. These formulations are fully described in the references and therefore will not be repeated. Since the thermoelectric problem only requires two degrees of freedom per node 
(temperature and voltage), the assembled FE matrix is:

$$
\left[\begin{array}{cc}
c_{1} \mathcal{K}^{T T}+c_{2} \mathcal{C}^{T T}+c_{3} \boldsymbol{M}^{T T} & c_{1} \mathcal{K}^{T V}+c_{2} \mathcal{C}^{T V} \\
c_{1} \mathcal{K}^{V T}+c_{2} \mathcal{C}^{V T} & c_{1} \mathcal{K}^{V V}
\end{array}\right]
$$

where $c_{1}, c_{2}, c_{3}$ are time integration parameters and $\mathcal{K}, \mathcal{C}, \boldsymbol{M}$ the tangent conductivity, capacity and thermal inertia matrices, respectively. Note that $\mathcal{K}$ is denominated stiffness matrix in the Continuum Mechanics community; however, for the thermoelectric problem it represents the conductivity matrix. Note also that the coupled matrix will not be symmetric, requiring a special algorithm for the inversion of the assembled matrix.

All terms in the assembled matrix are developed in [19]. In particular, the submatrices $\mathcal{C}^{V T}, \mathcal{C}^{T V}$ were incorporated in [7] to take into account $\tau_{j q}$, $\tau_{q j}$, respectively:

$$
\begin{aligned}
& \mathcal{C}_{A B}^{V T}=-\tau_{j q} \int_{\Omega}\left(\mathcal{B}_{A}\right)^{\mathrm{t}} \alpha \gamma \mathcal{B}_{B} \mathrm{~d} \Omega \\
& \mathcal{C}_{A B}^{T V}=-\tau_{q j} \int_{\Omega}\left(\mathcal{B}_{A}\right)^{\mathrm{t}} T_{B} \alpha \gamma \mathcal{B}_{B} \mathrm{~d} \Omega
\end{aligned}
$$

where $A, B$ denote two generic global FE nodes, $\Omega$ the domain and $\mathcal{B}$ the discretized gradient matrix.

The time-integration algorithm is regularized to avoid Gibbs phenomena with the procedure described in [19]. Since the FE formulation was intended to be complete, a three-dimensional isoparametric element is used, although due to geometry and boundary conditions the models are one-dimensional in the present work.

Finally, the FE formulation was implemented into the research code FEAP [20], from the University of California at Berkeley. This code provides several 
dummy routines (user elements) that can be used for the implementation of newly developed modular elements written in Fortran.

\section{Calibration of the numerical model: inverse problem}

Material properties are required for the numerical simulation of the experiment from [1], but some of these properties were not reported. In addition, the relaxation time is not considered in the experimental work ( $K$ is empirically fitted). Therefore, the present numerical model is calibrated with two steps:

1. A Sensitivity Analysis (SA) to identify the thin-film properties that are relevant for the hysteretic behavior

2. An identification IP to quantify these properties from a single experimental curve

\subsection{Sensitivity Analysis}

The objective of the SA is the determination of the relationships between the uncertainties for dependent and independent (or random) variables. The $\mathrm{SA}$ is a method for checking the quality of a given model; there are many available procedures to develop a SA and we apply the one from [21]. This procedure uses the concept of Standardized Regression Coefficients (SRC), whose absolute values provide a measure of the importance of each variable, $[22]$.

The dependent variable is a cost function defined as the quadratic differ-

ence between the voltage drops obtained from the experimental curve $\Delta V^{E X P}$ 
drawn in Figure 2 bottom, and from the FE simulation $\Delta V^{N U M}$ :

$$
f=\frac{1}{N} \sum_{i=1}^{N}\left(\Delta V_{i}^{E X P}-\Delta V_{i}^{N U M}\right)^{2}
$$

where $N$ is the number of measurement points or FE nodes.

The random variables are defined as the set $\left\{\alpha, \gamma, \kappa, c, \rho_{m}, \tau_{q}, \tau_{j q}\right\}$ and are assumed to be normally distributed: mean values given in Table 2 are obtained from [23], [1], and standard deviations are assumed to be $25 \%$. These deviations are higher than the usual $10 \%$ to take into account the worst-case scenario.

To reduce $\mathrm{CPU}$ cost and at the same time guarantee convergence, an optimized sample of size $m=1000$ was calculated by the procedure developed in [24]. According to this reference, the sample is of the Latin Hypercube type since the convergence is faster than the one related with random techniques.

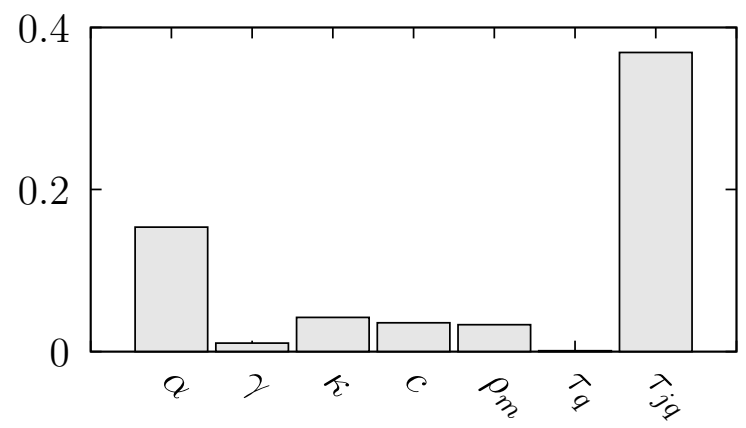

Figure 3: Standardized regression coefficients in absolute value for random variables (material properties).

Figure 3 shows the SRC's in absolute value obtained from the SA. As expected, the cost function is sensitive to the Seebeck coefficient $\alpha$ and particularly sensitive to the relaxation time $\tau_{j q}$. These facts agree with the 
theoretical assumptions made in Section 3.2: the loop shape and its diagonal slope depend on $\tau_{j q}$ and $\alpha$, respectively. The sensitivities of $\kappa, c, \rho_{m}$ are smaller but relevant, since the electric energy generated by the Seebeck effects depends on the thermal material properties as will be shown below. Finally, the sensitivities of $\gamma, \tau_{q}$ are not relevant at all, the first due to $\boldsymbol{j}=\mathbf{0}$. The second irrelevancy implies that the purely thermal viscosity is not present in this phenomenon, $\tau_{q} / t_{o b}<<1$. Consequently, these two random variables will not be considered in the following.

\subsection{Inverse problem}

The identification IP is directed to evaluate the magnitude of the random variables identified by the SA from a reduced set of data, in particular experimental loops $\Delta V-\Delta T$. Note that the material properties taken from the references are not used now, with the objective of characterizing the material without the need of a complete set of experiments. The IP is solved using the procedure described in [24], [25], defining: (i) a set of output variables (parametrization) introduced into the FE code to solve the direct problem, (ii) a cost function and (iii) a minimization method.

\section{Parametrization}

In the IP framework, the concept of model parametrization implies the definition of the possible solution through a set of parameters, that are the working variables and at the same time the IP output. The choice of the parametrization is often not obvious, a critical step in the problem setup. Here, according to the SA results from Figure 3, the output set is defined as $\left\{\alpha, \kappa, c, \rho_{m}, \tau_{j q}\right\}$. Starting from a wide range of these parameters given by 
the user, the optimization algorithm searches the optimal values, that must be very similar to the ones taken from the literature.

\section{Cost function}

The cost function (12) is redefined as:

$$
f^{L}=\log (f+\varepsilon)
$$

where $\varepsilon=10^{-16}$ is a very small non-dimensional value that ensures the

function existence when $f \rightarrow 0$. According to [26], this redefinition often increases the minimization algorithm convergence.

\section{Minimization}

A standard Genetic Algorithm (GA), see [27], is employed to minimize (13) and to obtain the IP output listed in the first column of Table 2. Other optimization techniques such as gradient-based algorithms could be applied, but according to [28] the GA guarantees convergence, whereas gradient-based algorithms strongly depend on the initial guess.

Table 1 first column lists the intrinsic GA parameters. The selected population size permits to find a global optimum with an adequate computational

\begin{tabular}{|c|c|}
\hline Parameter & Value \\
\hline Population size & 30 \\
Crossover ratio & 0.8 \\
Mutation ratio & 0.02 \\
Number of generations & 100 \\
\hline
\end{tabular}

Table 1: Parameter values for the genetic algorithm. 


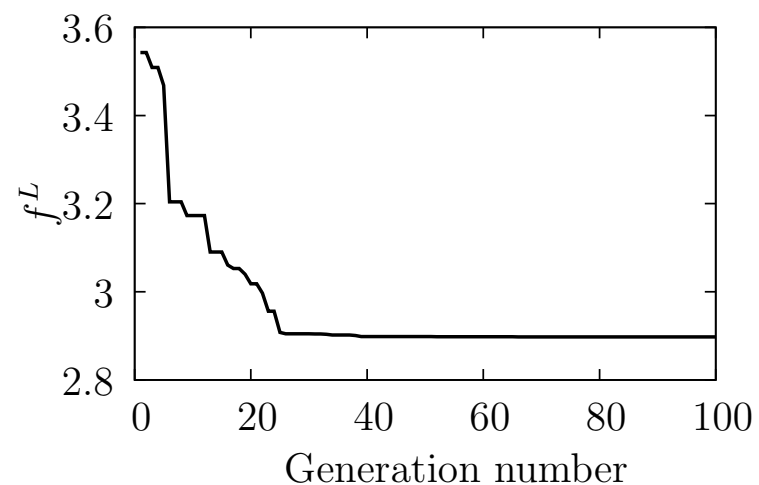

Figure 4: Genetic algorithm convergence. Cost function vs. number of generation.

cost. The mutation and crossover parameters are found by trial and error and inject genetic diversity, ensuring that the solution does not fall in a local minima. A large number of generations is chosen to warrant convergence.

\subsection{IP results}

Figure 4 shows the GA convergence to fit the experimental curve of Figure 5 top (see below), reached with approximately 50 generations and therefore evidencing that the GA parameters from Table 1 were correctly chosen. The IP is repeated ten times to ensure accurate results; the corresponding means and standard deviations are listed in Table 2 second and third columns. Standard deviations are less than $2 \%$, except for the most sensitive parameters $\alpha, \tau_{j q}$ with a $3 \%$.

The calculated $\kappa, c, \rho_{m}$ are very close to the experimental ones (fourth column). Also, the calculated $\alpha, \tau_{j q}$ can be compared with those empirically fitted in [1]. The relative errors between the IP and empirical results are approximately equal to the standard deviations obtained minimizing the problem with GA: the proposed FE-IP combination is suitable for the correct 
calibration of the material from a single experimental curve.

From the calculated parameters, FE and experimental responses are compared in Figures 5 for samples of Ti-doped $\mathrm{FeS}_{2}$ of the $\mathrm{n}$-type, in the top $\Delta V$ versus time and in the bottom $\Delta V$ versus $\Delta T$. The FE model (circles) correctly reproduces the experimental distribution; the only noticeable differences appear at the initial times, for which some error is due to the sharp discontinuity (from $\Delta T=0$ to $2[\mathrm{~K}]$ ) of the initial boundary conditions, see second term on the right side of (9). Smoother initial boundary conditions would fix the problem, but this error is not important for the targeted results.

In the same figure, $\mathrm{FE}$ distributions for two different $\tau_{j q}$ have also been plotted to study the influence of the relaxation time on the loop shape; as explained before the lower the relaxation the narrower loop, in particular there is no hysteresis if $\tau_{j q}=0$. Although no experimental comparisons are available, the distributions when $\tau_{j q}$ decreases imply that the error at initial times is mitigated. This fact is again due to the second term on the right side of (9): the smaller $\tau_{j q}$ the less influent this term is.

Figure 6 shows the same comparison but for a p-type Ti-doping; the agreement between experimental and numerical loops is again very good. Ten IP cases have been executed to recalculate the material properties, obtaining the same $\kappa, c, \rho_{m}$ but different $\alpha=55.5[\mu \mathrm{V} / \mathrm{K}], \tau_{j q}=30.6[\mathrm{~s}]$, probably due to a difference in the doping process. No comparison is given here since no values are reported. The Seebeck coefficient changes sign due to the ptype doping and its value is smaller, hinting the lower performance of this material. The value of $\tau_{j q}$ is about one third, reflecting the narrowness of the loop with respect to that of Figure 5. This reduction is also detrimental since 
from (9) it can be observed that the higher the relaxation time the higher the voltage drop.

The FE-IP combination is now used to characterize two new samples from [1], executing again ten IP's and obtaining values:

- for a PdS sample a calculated $\alpha=-350 \pm 32[\mu \mathrm{V} / \mathrm{K}]$, same order as the experimental $-300 \pm 40$ reported in [29]

- for an n-type $\mathrm{FeS}_{2}$ sample a calculated $\alpha=-18 \pm 3[\mu \mathrm{V} / \mathrm{K}]$ again close to the experimental $\alpha=-19$, measured in [1].

The calculated relaxation times for both samples are $\tau_{j q}=30,102[\mathrm{~s}]$, not measured in the references.

Finally, a more complete experiment from [1] for the second sample is numerically reproduced in Figure 7: a non-regular $\Delta T$ versus time signal is applied and the voltage drop is measured. Four pulses of different bandwidth and amplitude are prescribed (upper right) producing four loops (main figure). The objective of this experiment was to show that all loops have a common slope $\alpha$, making possible its measurement. To accurately replicate the experiment, a careful data reading with a smoothing technique of the slopes and amplitudes is necessary. Four different symbols are used to differentiate the four loops. The agreement between experimental and numerical results is again very good, not only for the loops but also for the distributions at the beginning and at the end of the process. 


\section{Design of experiments for the validation of the theoretical ex- planation}

The aim of this section is to present three numerical cases that could be experimentally performed in the future to further validate the theoretical

explanation for the hysteretic behavior. From the energy balance (1) and transport equations (5), and assuming $\boldsymbol{j}=\mathbf{0}, \tau_{q}=0$ as before, the following Poisson equation is obtained:

$$
\nabla^{2} V=-\alpha \frac{\rho_{m} c}{\kappa} \dot{T}-\tau_{j q} \alpha \frac{\rho_{m} c}{\kappa} \ddot{T}
$$

In (14), the sources (terms on the right side) depend on material properties $\alpha, \kappa, \rho_{m}, c$, on temperature derivatives $\dot{T}, \ddot{T}$ and on the relaxation time, $\tau_{j q}$; according to the proposed explanation:

$$
\tau_{j q}=0 \quad \text { or } \quad \ddot{T}=0 \quad \Rightarrow \quad \text { No hysteresis }
$$

As already verified, there will be no hysteretic behavior if $\tau_{j q}=0$. To check now the absence of hysteresis when the prescribed signal is so that $\ddot{T} \equiv \mathrm{d}^{2}(\Delta T) / \mathrm{d} t^{2}=0$, three numerical cases are performed in the following subsections. In all of them, the simulation is done with the FE, studying an n-type sample of Ti-doped $\mathrm{FeS}_{2}$ material.

\subsection{Case $\mathbf{A}$}

The signal is forced to have $\ddot{T}=0$ with a linear $T$ shown in Figure 8 top left. A completely linear response without any hysteresis is obtained when $\Delta V$ is plotted versus $\Delta T$ as in the top right, and its slope is the unique $\alpha$ value. Therefore, an alternative for the proper characterization of the Seebeck 
coefficient of these materials would be to apply a $\ddot{T}=0$ signal and use the classical relation $(7)$.

\subsection{Case $\mathbf{B}$}

In this case, the influence of the frequency is studied; two sinusoidal $T$ signals with the same amplitude but different frequencies $\omega=4 \pi / \hat{t}, 12 \pi / \hat{t}$ are prescribed as in Figure 8 middle left. The signal is applied up to an arbitrary time of $60[\mathrm{~s}]$. The middle right figure shows two recurrent and superimposed hysteretic ellipses for each of the periods. For both frequencies, hysteretic behaviors are observed, since $\tau_{j q} \neq 0$ and $\ddot{T} \neq 0$. Three interesting remarks can be made:

- At $\Delta T= \pm 1[\mathrm{~K}]$, the voltage drop for the two signals is equal, since for both $\ddot{T}=0$

- At $\Delta T=0[\mathrm{~K}]$ the difference between ellipse heights is maximum, since $\ddot{T}$ is also maximum

- The increase of voltage drop (ellipse height) is proportional to the signal frequency since $\ddot{T} \propto \omega^{2}$

The last remark confirms, as was argued in [5], that the influence of relaxation times is stronger for fast effects such as ultrasound waves. For these concentric ellipses, $\alpha$ could be obtained geometrically measuring their common slopes, as in [1].

\subsubsection{Case $\mathbf{C}$}

The influence of the $T$-signal amplitude is studied in this case. An exponentially increasing sinusoidal signal is applied, as in Figure 8 bottom 
left. The response, bottom right, is a growing spiral centered at the origin. The distance between two branches is proportional to the time between two consecutive signal periods.

Again the slope of the symmetry axis gives the Seebeck coefficient. Given that the same material is simulated, the three slopes of the right figures are equal, although they look different due to the different scale.

\section{Conclusions}

This work presents a theoretical explanation for the understanding of the hysteretic behavior in thin-film photovoltaic materials, using the extended non-equilibrium thermodynamics. It is concluded that the hysteresis depends on the relaxation time $\tau_{j q}$ and on the acceleration of the prescribed temperature $\ddot{T}$. The latter dependency could explain the strong influence of relaxation times on fast effects such as ultrasonic waves. Experimental cases from the literature have been simulated using a finite element formulation developed by the authors in previous publications, validating the theoretical explanation. Three numerical cases have been proposed and simulated to fully validate in the future our theoretical explanation with laboratory experiments.

In addition, an identification inverse problem has also been performed for the characterization of the Seebeck coefficient and of the relaxation time $\tau_{j q}$ along with other relevant material properties. The combination of the inverse

problem and of the finite element method permits a good characterization of thin-film material properties from a single experimental curve. 


\section{Acknowledgments}

This research was partially supported by the grants CSD2008-00037 Canfranc Underground Physics, Excelencia Junta Andalucía P08-TEP-03641 and Polytechnic University of Valencia under programs PAID 02-11-1828 and $05-10-2674$.

\section{References}

\section{References}

[1] I.J. Ferrer, P. Díaz-Chao, A. Pascual, and C. Sánchez. Hysteresis-like behaviour of the thermoelectric voltage in photovoltaic materials. Thin Solid Films, 511-512:177-181, 2006.

[2] D. Galushko, N. Ermakov, M. Karpovski, A. Palevski, J.S. Ishay, and D.J. Bergman. Electrical, thermoelectric and thermophysical properties of hornet cuticle. Semiconductor Science and Technology, 20:286-289, 2005.

[3] M. Plotkin, I. Hod, A. Zaban, S.A. Boden, D.M. Bagnall, D. Galushko, and D.J. Bergman. Solar energy harvesting in the epicuticle of the oriental hornet (Vespa Orientalis). Naturwissenschaften, 97:1067-1076, 2010.

[4] A.R. Varrenti, C. Zhou, A.G. Klock, S.H. Chyung, J. Long, S.O. Memik, and M. Grayson. Thermal sensing with lithographically patterned bimetallic thin-film thermocouples. IEEE Electron Device Letters, 32:818-820, 2011. 
[5] D. Jou and G. Lebon. Extended Irreversible Thermodynamics. SpringerVerlag Berlin Heidelberg, 1996.

[6] E. Hernández-Lemus and E. Orgaz. Hysteresis in nonequilibrium steady states: the role of dissipative couplings. Revista Mexicana de Física, 48:38-45, 2002.

[7] R. Palma. Thermodynamic Formulation for Non-linear Finite Element Applied to Multicoupled Materials. PhD thesis, University of Granada, 2012.

[8] S.R. de Groot and P. Mazur. Non-equilibrium Thermodynamics. Dover, 1984.

[9] R.Y. Nuwayhid, F. Moukalled, and N. Noueihed. On entropy generation in thermoelectric devices. Energy Conversion and Management, 41:891914, 2000 .

[10] H.B. Callen. Thermodynamics and an Introduction to Thermostatistics. John Wiley and Sons, Inc, 1985.

[11] D.M. Rowe, editor. CRC Handbook of Thermoelectrics. CRC Press, 1995.

[12] J.E. Llebot, D. Jou, and J. Casas-Vázquez. A thermodynamic approach to heat and electric conduction in solids. Physica, 121(A):552-562, 1983.

[13] C. Cattaneo. On heat conduction (in Italian). Atti Seminario Mat. Fis. University Modena, 3:83-1013, 1938. 
[14] H. Rahideh, P. Malekzadeh, and M.R. Golbahar. Heat conduction analysis of multi-layered FGMs considering the finite heat wave speed. Energy Conversion and Management, 55:14-19, 2012.

[15] P. Drude. On electrical theory for metals and on galvanomagnetic and thermomagnetic effects (in German). Annalen der Physik, 308:369, 1900.

[16] A. Rodríguez, D. Astrain, and A. Martínez. Study of thermoelectric systems applied to electric power generation. Energy Conversion and Management, 50:1236-1243, 2009.

[17] J.L. Pérez-Aparicio, R.L. Taylor, and D. Gavela. Finite element analysis of nonlinear fully coupled thermoelectric materials. Computational Mechanics, 40:35-45, 2007.

[18] J.L. Pérez-Aparicio, R. Palma, and R.L. Taylor. Finite element analysis and material sensitivity of Peltier thermoelectric cells coolers. International Journal of Heat and Mass Transfer, 55:1363-1374, 2012.

[19] R. Palma, J.L. Pérez-Aparicio, and R.L. Taylor. Non-linear finite element formulation applied to thermoelectric materials under hyperbolic heat conduction model. Computer Method in Applied Mechanics and Engineering, 213:93-103, 2012.

[20] R.L. Taylor. FEAP A Finite Element Analysis Program: User Manual. University of California, Berkeley, 2010. http://www.ce.berkeley.edu/feap.

[21] P. Hoes, M. Trcka, J.L.M. Hensen, and B.H. Bonnema. Investigating the potential of a novel low-energy house concept with hybrid adaptable 
thermal storage. Energy Conversion and Management, 52:2442-2447, 2011.

[22] I.P. Koronaki, E. Rogdakis, and T. Kakatsiou. Thermodynamic analysis of an open cycle solid desiccant cooling system using artificial neural network. Energy Conversion and Management, 60:152-160, 2012.

[23] K. Kato, Y. Okamoto, J. Morimoto, and T. Miyakawa. The thermoelectric properties of $\mathrm{FeS}_{2}$. Journal of Materials Science Letters, 16:914-916, 1997.

[24] R. Palma, G. Rus, and R. Gallego. Probabilistic inverse problem and system uncertainties for damage detection in piezoelectrics. Mechanics of Materials, 41:1000-1016, 2009.

[25] J. Zhao, Y. Song, W. Lam, W. Liu, Y. Liu, Y. Zhang, and D. Wang. Solar radiation transfer and performance analysis of an optimum photovoltaic/thermal system. Energy Conversion and Management, 52:1343$1353,2011$.

[26] R. Gallego and G. Rus. Identification of cracks and cavities using the topological sensitivity boundary integral equation. Computational Mechanics, 33:154-163, 2004.

[27] O.E. Canyurt and H.K. Ozturk. Three different applications of genetic algorithm (GA) search techniques on oil demand estimation. Energy Conversion and Management, 47:3138-3148, 2006.

[28] G. Rus, R. Palma, and J.L. Pérez-Aparicio. Optimal measurement setup 
for damage detection in piezoelectric plates. International Journal of Engineering Science, 47:554-572, 2009.

[29] I.J. Ferrer, P. Díaz-Chao, A. Pascual, and C. Sánchez. An investigation on palladium sulphide (PdS) thin films as a photovoltaic material. Thin Solid Films, 515:5783-5786, 2007. 


\begin{tabular}{|c|c|c|c|l|}
\hline $\begin{array}{c}\text { Para- } \\
\text { meter }\end{array}$ & $\begin{array}{c}\text { Mean } \\
\text { IP }\end{array}$ & $\begin{array}{c}\text { Standard } \\
\text { deviation }\end{array}$ & $\begin{array}{c}\text { Experi- } \\
\text { mental }\end{array}$ & \\
\hline$\alpha$ & -68 & 3 & -65.8 & {$[\mu \mathrm{V} / \mathrm{K}]$} \\
$\kappa$ & 1.66 & 1.8 & 2 & {$[\mathrm{~W} / \mathrm{mK}]$} \\
$c$ & 534 & 1.9 & 547 & {$[\mathrm{~J} / \mathrm{KgK}]$} \\
$\rho_{m}$ & 5328 & 1.3 & 4900 & {$\left[\mathrm{Kg} / \mathrm{m}^{3}\right]$} \\
$\tau_{j q}$ & 93 & 3 & 93 & {$[\mathrm{~s}]$} \\
\hline
\end{tabular}

Table 2: Means and standard deviations obtained solving ten times the inverse problem for an n-type sample of Ti-doped $\mathrm{FeS}_{2}$. Experimental values taken from [23] except $\alpha, \tau_{j q}$ empirically found in [1]. 

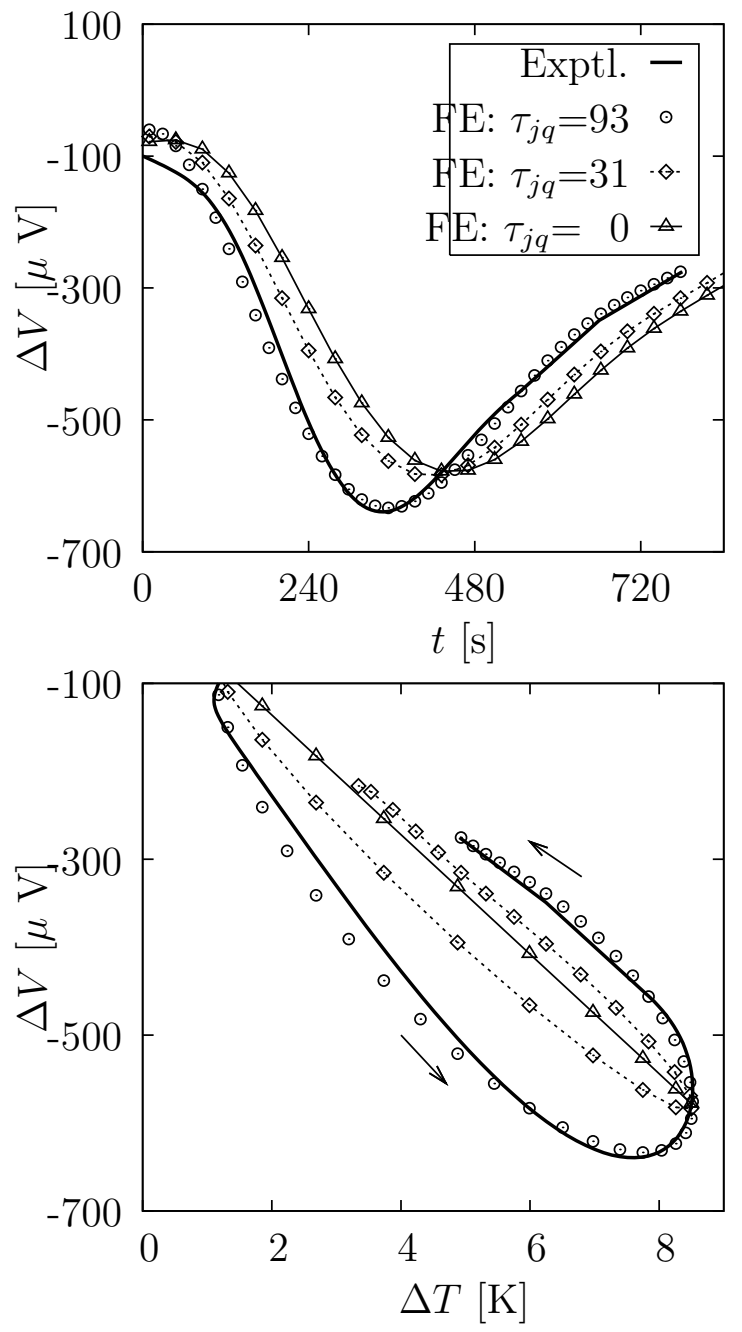

Figure 5: Experimental (thick line) and finite element (symbols) results for three relaxation times, n-type Ti-doped $\mathrm{FeS}_{2}$. Top: voltage drop vs. time, bottom: idem vs. prescribed temperature difference. 


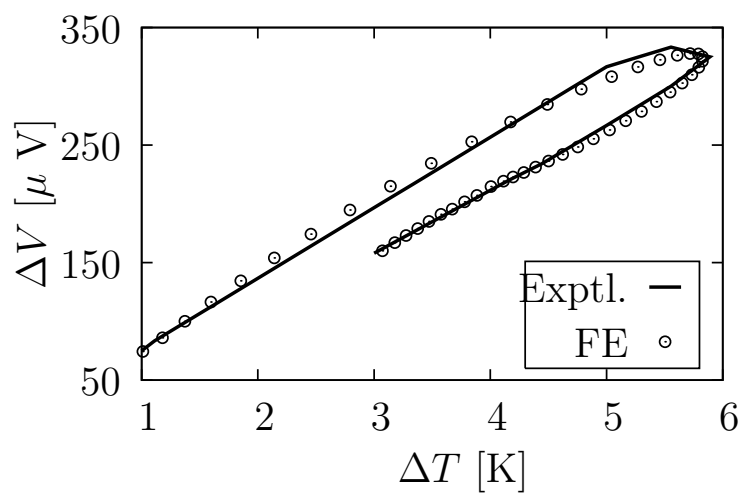

Figure 6: Experimental (solid line) and finite element (circles) results for a p-type sample of Ti-doped $\mathrm{FeS}_{2}$. Measured voltage drop vs. applied temperature difference.

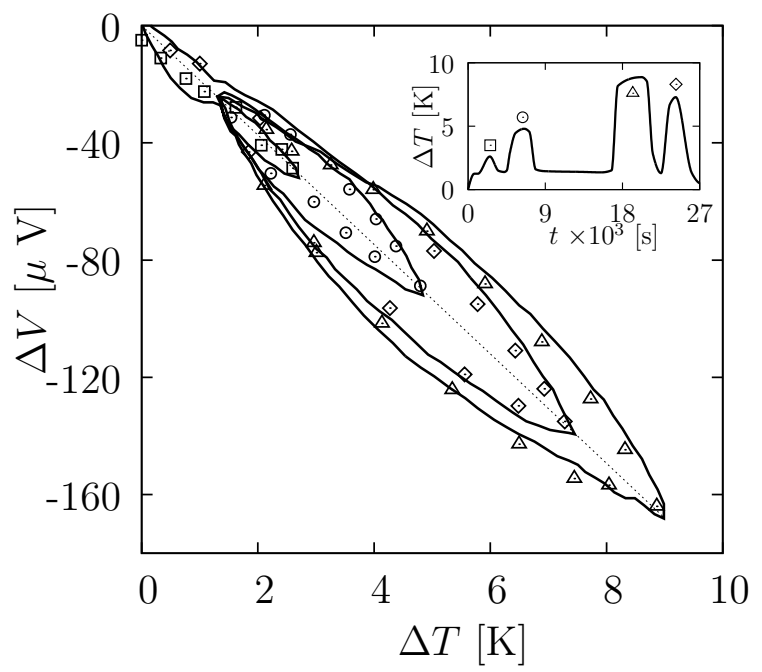

Figure 7: Experimental (solid line) and finite element (symbol for each peak) results for n-type sample of $\mathrm{FeS}_{2}$. Measured voltage drop vs. applied temperature difference for four different peaks. 

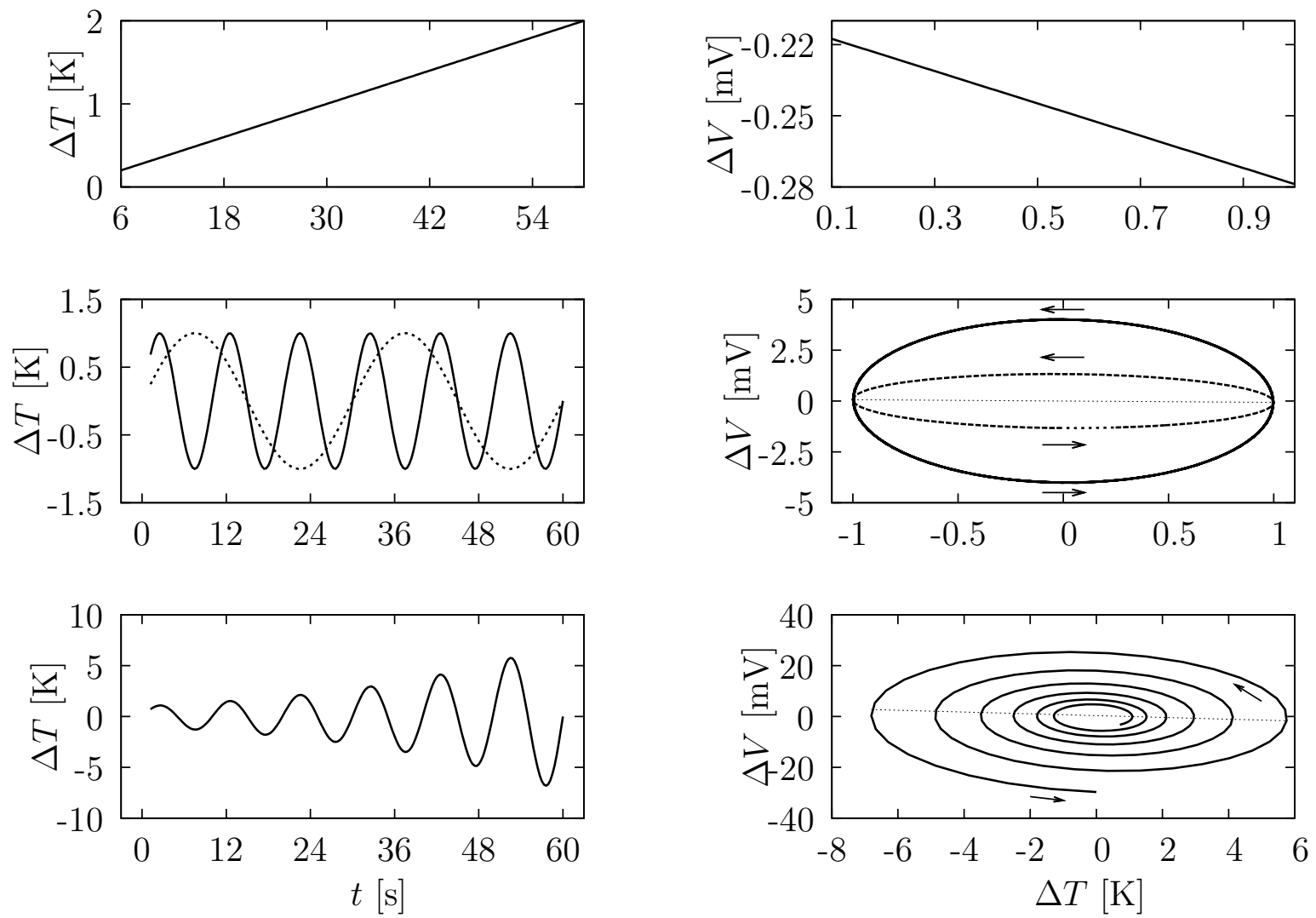

Figure 8: Proposed numerical experiment temperature increment signals vs. time (left) and resulting voltage drop vs. difference temperature (right) for an n-type sample of Ti-doped $\mathrm{FeS}_{2}$. Applied signals: linear (top), sinusoidals (middle) and exponentially increasing (bottom). For middle figure, frequencies $\omega=4 \pi / \hat{t}$ continuous line, $12 \pi / \hat{t}$, dashed. Only finite element results shown. 


\section{Response to Reviewer \#2:}

The authors greatly appreciate the reviewer's efforts on this manuscript to provide valuable comments and constructive critiques. These observations have guided us in the revision of the manuscript. We have carefully followed the reviewer's suggestions and have made the corresponding changes in the new version. Detailed responses to each issue pointed out by the reviewer (in italics) are commented underneath (in regular typography):

Figures 2,5 and 6 contains some displacements.

Response: All displacements will automatically be corrected when the final two-columns format of this journal is retrieved. The figures were optimized with the software "gnuplot " for two-columns, it was the required compilation for one-column that is distorting the figures.
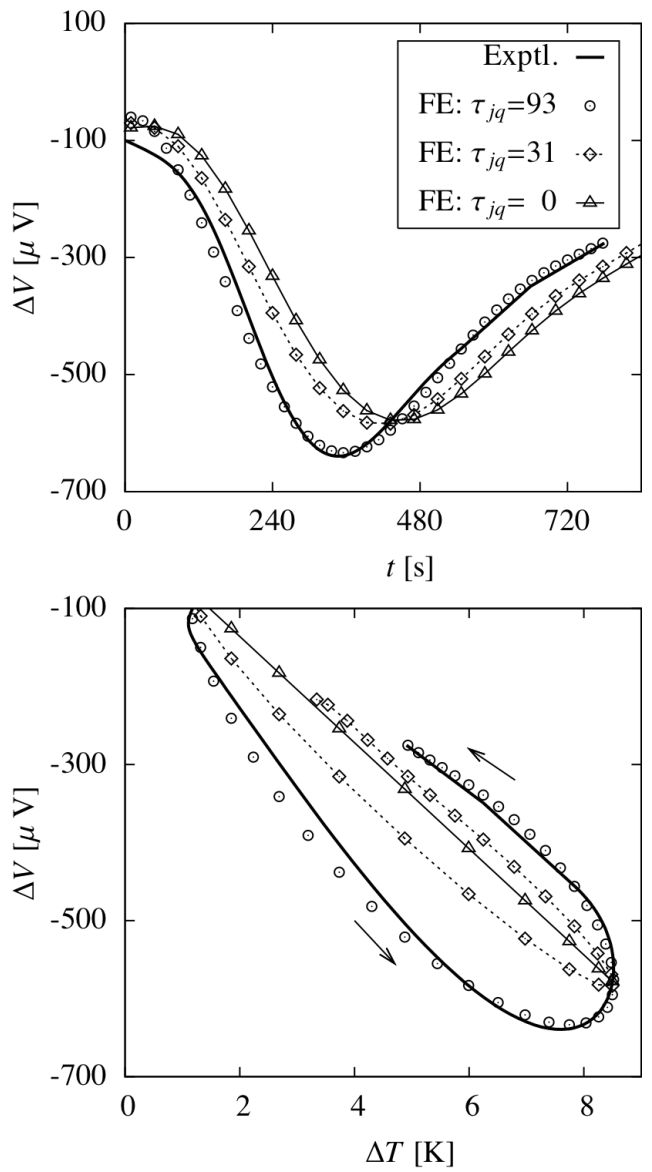

Figure 5: Experimental (thick line) and finite element (symbols) results for three relaxation times, n-type Ti-doped $\mathrm{FeS}_{2}$. Top: voltage drop vs. time, bottom: idem vs. prescribed temperature difference. 